\title{
Una aproximación a los postgrados e investigación en psicología paraguaya
}

\author{
Norma B. Coppari \\ Universidad Católica de Asunción \\ Universidad Nacional de Asunción
}

\begin{abstract}
Resumen
El derrotero histórico del país, en materia de desarrollo científico y tecnológico, muestra ausencia o peso insignificante, en las políticas de los gobiernos. En particular, los postgrados en Psicología han sido esporádicos, de cuestionable calidad y de escasa trascendencia en el contexto académico y profesional. Se requiere la implementación y fortalecimiento de políticas educativas de calidad y sistemáticas en educación superior y en especial de la formación científica en básica, aplicada y tecnológica. Se destacan los Tratados del MERCOSUR en formación, la creación de ANEAES (2003) para los procesos de acreditación de la calidad, y la labor de la SPPs para la Ley de Colegiatura. El siglo XXI espera por una Psicología Paraguaya fortalecida en su perfil científico-practicante local frente a desafíos regionales y mundiales.
\end{abstract}

Palabras Claves: postgrados; investigación; psicología.

\begin{abstract}
An approach to graduate and research in paraguayan psychology. The historic route of the country, in terms of scientific and technological development, shows the insignificant weight in the policies of governments. In particular, the graduate degrees in psychology have been sporadic, of questionable quality and of little importance in the academic and profesional context. It requires the implementation and strengthening of educational policies and consistency in the quality of higher education, especially basic, applied and technological research. Noteworthy are the treaties in the formation of MERCOSUR, the creation of ANEAES (2003) for the process of quality accreditation, and the work of the SPPs for the Education Act. The twentyfirst century brings the expectation of a paraguayan psychology stronger in its local scientific and practical profile, and able to confront the regional and global challenges.
\end{abstract}

Keywords: postgraduate; research; psychology.

\section{Resumo}

Uma aproximação aos programas de pesquisa e pós-graduação na psicologia paraguaia. O traçado histórico do país, em termos de desenvolvimento científico e tecnológico, revela um peso insignificante ou inexistente por parte das políticas governamentais. Em particular, os programas de pós-graduação em psicologia têm sido esporádicos, de qualidade questionável e com pouca relevância no âmbito acadêmico e profissional. Faz-se imprescindível a implementação e o fortalecimento de políticas educativas sistemáticas e de qualidade no ensino superior, além da formação científica em pesquisa básica, aplicada e tecnológica. Destacam-se os tratados do MERCOSUL na formação e criação do ANEAES (2003) para os processos de creditação da qualidade, e o trabalho da SPPs para a Lei de Ensino. O século XXI traz a expectativa de uma psicologia paraguaia fortalecida em seu perfil científico-prático local, e apta a enfrentar os desafios regionais e mundiais.

Palavras-chave: pesquisa; pós-graduação; psicologia.

\section{Contexto General}

Paraguay ocupa una superficie total mediterránea de 406.752 $\mathrm{Km}^{2}$ y tiene 7.000.000 de habitantes aproximadamente. Está dividido por el río del mismo nombre en dos grandes regiones: la Oriental y la Occidental o Chaco, que presentan factores físicos, climáticos y administrativos propios. En la región
Oriental, que ocupa un 39\% del territorio se asienta la mayor parte de la población, esto es, un $98 \%$ de los habitantes, y en igual porcentaje, su rubro potencial mas importante es de carácter rural agropecuario.

Siguiendo el derrotero histórico del país, en materia de desarrollo científico y tecnológico, es posible afirmar su ausencia 
o peso insignificante, en las políticas de los gobiernos durante más de 70 años del siglo XX. Los factores de este atraso son diversos. Las dos grandes guerras, la de la Triple Alianza (1864-1870) y la del Chaco (1932-1935) sumieron al país en necesidades económicas, de reconstrucción y de inestabilidad política, postergando y rezagando el fomento en la educación, la cultura, la investigación científica, la integración entre ciencia y tecnología y la producción de bienes y servicios.

Si bien, la primera institución de formación superior, la Universidad Nacional de Asunción (UNA), cumplió ya 100 años desde su creación, en pleno período de entre-guerras (1889-2009), la misma ha tenido, en su carácter de institución pública, infraestructura y presupuestos precarios, nula inversión en ciencia y tecnología en casi toda su existencia. Se ha enfatizado la docencia, bajo la forma de transmisión y repetición de conocimiento más que en la generación del mismo, o en su innovación. En la década del 1960 se crea la primera universidad privada, dependiente de la Conferencia Episcopal Paraguaya, la Universidad Católica "Nuestra Señora de la Asunción” (UCA), mas el modelado de aquel patrón persiste en las instituciones de educación superior, tanto, nacionales como privadas, de las décadas del 1970 y 1980, dándose un crecimiento desmedido (casi 60\%), en la apertura de nuevas universidades a partir del 1980, 1990 y 2000, por cierto, de dudosa calidad educativa.

A diferencia de los demás países de la región que buscaron abrirse a la cooperación internacional para desarrollar ciencia y tecnología aplicada a la economía en los procesos de industrialización, Paraguay se aíslo políticamente durante la dictadura, y se detuvo en gran medida, en un sistema de producción agraria predominantemente familiar. La escasa investigación que se venía realizando se enfocaba casi totalmente en los problemas de salud pública y medicina. Pero en ningún caso se sistematizó la investigación como resultado de la aplicación de políticas públicas específicas y explícitas.

A pesar de ese contexto, la cooperación internacional, en materia de financiación no reembolsable como también, en el rubro de asistencia técnica y científica, fue vital para el establecimiento y equipamiento de las instituciones públicas de planificación económica y social y las que desarrollaban actividades científicas y tecnológicas (incluida la UNA). La cooperación se dio con mayor énfasis en la década de 1960 y 1970, con la fuerte participación de los organismos del sistema de las Naciones Unidas (Consejo Nacional de Ciencia y Tecnología - CONACYT, 2002).

No obstante, la cooperación internacional y el crecimiento de las universidades, poco o nada se hizo para organizar y fomentar la investigación en todos sus niveles (básica, aplicada y tecnológica), en las diversas disciplinas académicas, perdiéndose valiosas oportunidades como la de incentivar el desarrollo de las ciencias y tecnologías vinculadas a la construcción de centrales hidroeléctricas (Acaray, Itaipu e Yacyretá), durante las décadas de 1970 y 1980. Las universidades omitieron casi totalmente estos aspectos en sus actividades académicas.

En lo institucional, el sector de ciencia y tecnología actuó siempre como un conglomerado disperso de instituciones sin un organismo rector de políticas. Los institutos dependientes de los Ministerios se regían por las políticas de éstos, cuando existían. Las universidades se dedicaban, con raras excepciones, a la formación de profesionales sin ninguna o poca actividad en el área de investigación. En realidad, no existía un sistema de ciencia y tecnología, en el sentido estricto del término, sino un conglomerado de instituciones y unidades orientadas cada una en un sentido, en ciertas ocasiones totalmente divergente.

La organización institucional del sector solamente se dio después del ingreso al proceso democrático (desde 1989). La elaboración y aprobación de un marco legal llevó casi una década de discusiones entre diversas instituciones y actores involucrados (Ministerios, universidades, profesionales, gremios industriales, institutos de investigación y el Congreso Nacional). Finalmente, en 1997, se promulgó la Ley 1028, “General de Ciencia y Tecnología”, a través de la cual se creó el CONACYT.

Según la Ley de creación del CONACYT, su objetivo principal es dirigir, coordinar y evaluar las actividades del Sistema Nacional de Ciencia y Tecnología, así como del Sistema Nacional de Calidad. Para cumplir dichos objetivos, el CONACYT cuenta con atribuciones legales para formular y proponer al gobierno las políticas de Ciencia, Tecnología, Innovación y Calidad del país, impulsar la capacitación altamente calificada de recursos humanos y apoyar financieramente a los proyectos de investigación.

Como instrumento de financiamiento de los programas de apoyo al sector, la misma Ley creó el Fondo Nacional de Ciencia y Tecnología (FONACYT), otorgando al CONACYT la atribución de administrar los recursos del mismo (CONACYT, 2002).

En general, y en lo particular del ámbito que nos interesa introducir, el de la ciencia psicológica, se aprecia de todo lo citado, que las actividades científicas y tecnológicas en el país no han sido notables en la historia, tampoco su tratamiento ha tenido un peso gravitante en las políticas públicas - salvo excepciones a mediados del siglo XIX. La consecuencia actual es la brecha de conocimiento no solamente con los países más desarrollados del mundo, sino también con la mayor parte de los demás países sudamericanos, y mas, recientemente, con el MERCOSUR.

El hecho de que exista ese rezago comparativo en la generación, profundización y aplicación de conocimientos científicos y tecnológicos en el país produce no solamente un efecto directo en si (por ejemplo, el escasísimo desarrollo de las ciencias básicas), en el sentido de llevar al país a los últimos lugares en cuanto a producción científica, sino también afecta directamente a la capacidad de innovación productiva, la competitividad de la producción nacional, el nivel y la calidad de vida de la población, la productividad y la capacidad de producción de bienes exportables con alto valor agregado. En la actualidad, el hecho de dejar un lugar marginal para la Investigación y Desarrollo (I+D) constituye un obstáculo para el crecimiento cultural, social y económico de los países (CONACYT, 2002).

El concepto de desarrollo ha cambiado notablemente en la actualidad, puesto que el mismo no solamente implica el crecimiento de indicadores económicos o sociales, sino también implica los conceptos de sustentabilidad, justicia social y eficiencia. Esta nueva visión de desarrollo se basa en un tejido estructural con alto contenido de conocimiento. 
Por consiguiente, para revertir la situación actual del país y permitir el desarrollo siguiendo las concepciones mencionadas, dentro de un marco de coordinación y articulación de acciones, es necesario trazar una política nacional que, al mismo tiempo parta de la realidad nacional, promueva la solución de problemas del presente y trace ejes de acción futura, con base en análisis prospectivos.

La Ley de Ciencia y Tecnología establece que el desarrollo científico y tecnológico del país estará orientado por políticas y programas específicos impulsados por el sector público, pero en concertación con el sector privado (Art. 3 de la Ley 1028/97). Establece, asimismo, que las políticas de largo plazo contendrán las pautas y estrategias generales para el desarrollo del sector y que deberán estar en concordancia con la política de desarrollo económico y social (Art. 3 y 7 de la Ley 1028/97).

Por todo lo expuesto, se entiende que la formulación de la Política Nacional de Ciencia y Tecnología: Objetivos y Estrategias tiene como fundamentos los siguientes aspectos: a) la situación actual de globalización económica exige un desarrollo económico y social que tenga como base la innovación productiva, lo cual implica la existencia de condiciones favorables para la generación, mejoramiento, transferencia, aplicación o adaptación de conocimientos científicos y tecnológicos; b) el escaso desarrollo de las ciencias básicas en el país es un impedimento para impulsar proyectos de investigación con potencial de éxito y de rigor científico; c) el CONACYT posee la atribución otorgada por Ley para formular y proponer la Política Nacional de Ciencia y Tecnología, en concordancia con la política de desarrollo económico y social; d) el Gobierno Nacional ha aprobado un Plan Estratégico Económico y Social, de cumplimiento obligatorio, en el cual se manifiesta la importancia de la CyT y se le da un lugar destacado (CONACYT, 2002).

La presente política tiene como alcance, la orientación de la investigación fundamental o básica, la investigación aplicada y el desarrollo de productos en el país, que sean llevadas a cabo mediante los recursos disponibles para el CONACYT, en todo el territorio nacional y en el contexto del marco legal vigente en la materia, en el horizonte de los próximos 5 años. Asimismo, tendrá un carácter indicativo para las actividades y servicios científicos y tecnológicos que se desarrollen en organismos e instituciones públicas, privadas y de la sociedad civil. Con la misma, se buscará racionalizar los recursos disponibles en el país y los que provengan de la cooperación internacional (CONACYT, 2002).

\section{La investigación en el ámbito universitario: desarrollo versus improductividad}

El contexto general esbozado en el apartado anterior, se refleja plenamente en los ámbitos particulares de las universidades más importantes de nuestro país, tanto nacionales como privadas.

Bajo el titulo del presente encabezado nos cupo hace unos años realizar unas investigaciones (Coppari, 1997a, 1997b, 2002), sobre la materia, aplicando para ello el Modelo de Evaluación Educativa: Contexto - Insumo - Proceso - Producto, resumido en la siglas CIPP (Stufflebeam et al., 1981), a la evaluación del estado de la Investigación, de la Docencia y de los Servicios, como fines Universitarios, en el contexto especifico de la Carrera de Psicología de la Universidad Católica. En ambos estudios se analizaron los cuatro indicadores propuestos y se obtuvieron estas conclusiones.

Contexto. Ausencia o inoperancia del Órgano o Entidad institucional, que proyecte, organice, ordene, norme, asesore, evalúe y promueva investigación en su formación y ejecución.

Consecuencia de lo anterior: es la carencia de información para elaborar una Política General de objetivos, prioridades, criterios, áreas de formación, conocimiento y líneas de investigación a trabajar.

La Investigación no es valorada como función sustantiva y prioritaria en el ámbito universitario, y mas especifico, en Psicología, no se la aprecia como ciencia, prima como profesión, y en general, se aprecia, la falta de fortaleza de la investigación en todos los ámbitos de la sociedad.

Desconocimiento y poca confianza en que contribuya al mejoramiento de los otros fines universitarios: Docencia, Extensión y Difusión.

Insignificante tradición en formación de recursos humanos para la investigación, ausencia del perfil docente-investigador, prima la docencia como transmisión de conocimiento. No existe carrera, ni escalafón para la investigación.

La investigación cuando se da, es decidida por intereses y posibilidades personales del investigador. Lo que conlleva, a fragmentación e individualización de la tarea.

Factores como los precedentes determinan a su vez, la incomunicación entre investigadores e imposibilidad de formar masa crítica estudiantil, considerando que en el futuro, ellos son materia prima esencial para formación de recursos humanos, urge capacitarlos temprano en investigación científica psicológica, carente de ellos.

Evidentes desvinculaciones entre Docencia, Extensión y Difusión dentro de la Disciplina Psicológica.

Finalmente, a este nivel de análisis, es claro y notorio que la investigación en Psicología no se desarrolla según un plan trazado para la consecución de objetivos preestablecidos, si se da es asistematica y azarosa.

Insumo. Se observa carencia casi absoluta de presupuesto, recursos humanos calificados, materiales, laboratorios, equipo e instalaciones específicas para su realización.

Caso de darse, predomina un comportamiento patrimonialista de los administradores sobre los recursos existentes.

Falta especificación de mecanismos para su instrumentación; carencia o predominio de programas obsoletos en la formación de recursos humanos para su realización.

Escaso fomento de enlaces institucionales con organismos nacionales e internacionales que apoyen el financiamiento, intercambio y promoción conjunta de investigación en sus diferentes niveles (Básica, Aplicada, Tecnológica, etc.) y abordajes metodológicos.

La inexistencia de un sistema adecuado de calificación y promoción del personal científico, conlleva la urgente 
elaboración de criterios objetivos y actualizados para la evaluación de docentes-investigadores.

La marginación de la investigación científica como parte fundamental de la actividad universitaria Si se desarrolla investigación en Psicología, pese a todas las carencias citadas, es porque la llevan adelante los pioneros esfuerzos de contadas individualidades docentes.

La carencia de apoyo gubernamental y privado a actividades de investigación, como la falta de fondos para las actividades científicas y la carencia de estímulo gubernamental a actividades de investigación en las universidades nacionales, más notoria en las privadas.

La dependencia externa en materia de ciencia, tecnología e innovación.

La elaboración de presupuestos nacionales "Base cero" sin adecuada evaluación y control.

La existencia de confusión formal y conceptual con respecto a la investigación y los investigadores, aún entre formadores del ámbito universitario.

La escasa vinculación entre la educación básica, media y superior, y la ciencia y la tecnología.

Proceso. Déficits importantes en la creación de políticas globales de evaluación e información sistematizada sobre las investigaciones terminadas o en curso (caso concreto de las Tesis y tesinas en Psicología, o de las producidas por materias responsables de generar investigación).

Déficit de investigación sobre los factores que inciden en las diferentes etapas del desarrollo de las mismas y obstaculizan el normal desarrollo y productividad.

La falta de valoración social y gubernamental a la producción del conocimiento y la débil vinculación internacional de los sectores vinculados a la producción científica - tecnológica.

Indigencia en bancos de datos y dificultad para el fácil acceso a información sobre la evolución del campo psicológico en investigación básica, aplicada y tecnológica; investigadores involucrados; recursos económicos y apoyos disponibles (esto en plena era de la información computarizada, servicios de Internet, Bibliotecas y hemerotecas virtuales, etc.).

Carencia de mecanismos y criterios operacionales para la evaluación periódica y permanente de la limitada producción, en términos institucionales, de la investigación por áreas temáticas, conocimiento en general, líneas y modalidades metodológicas, y de la labor y calificación de los investigadores.

La falta de vinculación y cooperación entre las escasas instituciones universitarias que hacen ciencia psicológica, tanto del sector publico como privado.

Producto. Es notorio el insignificante porcentaje terminal en la publicación de trabajos de investigación, no existen revistas científicas periódicas para la difusión de dichos productos a nivel universitario o de asociaciones profesionales.

Dándose las posibilidades de difusión, no raras veces, para aquellos investigadores que no se caracterizan precisamente, por la calidad de su producción, en detrimento de otros, que atesoran en el anonimato productos mucho más valiosos en sus aportes a nuestro campo.
Por otro lado, la investigación psicológica se ha limitado, cuando mucho a replicar o adaptar tecnologías, temas y metodología.

No se ha desarrollado la investigación original, de punta, o de vanguardia que se requiere para avanzar, desarrollar y revolucionar la disciplina psicológica; en términos más precisos, no se produce conocimiento nuevo u original.

La carencia de centros de documentación adecuados para acceder al conocimiento científico existente. La producción científica local no está sistematizada ni difundida.

Volviendo al contexto macro que nos determina, las observaciones logradas a nivel micro, en el campo específico de la ciencia psicológica, evidencian actualmente, muy escaso cambio en la situación estudiada, y generalización de los resultados reportados con antelación, a otros ámbitos distintos de la Psicología (Coppari, 1997a, 1997b, 2002), en cuanto al papel de la investigación científica y tecnológica a nivel de formación y promoción universitaria.

No existe a nivel macro y micro académico, una clara valoración de los alcances de la ciencia y tecnología en Psicología, lo que hace difícil la formación y promoción de actividades científicas y tecnológicas, y el consecuente impacto constructivo en el progreso de las disciplinas específicas en general, y de sus beneficios para la sociedad toda.

No termina de instalarse, en las esferas universitarias, como nicho propio de estos temas, una fructífera discusión sobre la importancia de la ciencia, la tecnología y la innovación en Psicología y otras ciencias, como motores del desarrollo material de país, pero si se considera que esta concepción debe ser asumida por la sociedad paraguaya para impulsar un salto cualitativo hacia un nuevo estilo de desarrollo productivo y búsqueda del bienestar.

Las críticas y clamores sobre la urgencia y pertinencia de reforma de las instituciones educativas a nivel básico, medio y superior, se ven frecuentemente enfrentadas con obstáculos en el plano político y legislativo, tal son los casos de los Proyectos de Ley de la Educación Superior y de Colegiatura Profesional impulsada por la SPPs, que siguen sin aprobación a la fecha.

Recientemente, la creación de la Agencia Nacional de Evaluación y Acreditación de la Educación Superior-ANEAES (2003) despierta mucha expectativa positiva, en tanto, se constituye en órgano auditor de la calidad educativa conforme a estándares internacionales, lo que a corto y largo plazo, llevara a que los procesos de acreditación, evalúen, valoren y certifiquen a las instituciones que reúnan aquellos requisitos mejorando el nivel de la educación superior del país. A la fecha, dificultades presupuestarias y de recursos humanos hacen que su necesario protagonismo no se evidencie.

No obstante, a nivel discursivo de los principales actores locales, se considera que la formación y promoción de la investigación científica y tecnológica en Psicología y otras ciencias, contribuirá a materializar el cambio deseado, para lo cual se enlistan a menudo, en ese mismo discurso, fortalezas, tales como: a) el proceso de construcción creciente de una masa crítica intelectual en el país y, mas específicamente, en el ámbito académico de la Psicología; b) el establecimiento de 
mecanismos legales de apoyo a la investigación (CONACYT, FONACYT, otros.); c) el fortalecimiento en los procesos de formación de recursos humanos capacitados e instalación de infraestructura para la investigación en el ámbito universitario; d) la creación y funcionamiento de centros de investigación extra-universitarios en el país; e) la valoración y premiación internacional de trabajos científicos realizados en el país; f) un mayor reconocimiento de la necesidad de fortalecer la formación y producción científica en las universidades nacionales y privadas; g) la creación de un Organismo Nacional de Protección Intelectual; h) el establecimiento de "Presupuesto con base cero" para el financiamiento de las actividades en la función pública.

A partir de estos indicadores positivos se percibe, a nivel macro, que existe capacidad para incrementar la débil producción científica existente, pero la misma necesita de condiciones más adecuadas que se pueden lograr mediante un estímulo sostenido de los sectores gubernamental y privado a las actividades que conducen a la creación de conocimientos y a la innovación tecnológica (Duarte Masi, 2005).

Los datos estadísticos obtenidos (CONACYT, 2010), actualizados al año 2008, reflejan esta situación a la fecha: Paraguay presenta uno de los PIB más bajos (16.920 millones de dólares), de la región latinoamericana (Tabla 1), y está entre los países que menos invierte en $\mathrm{I}+\mathrm{D}: 0,06 \%$, hallándose muy por debajo de America Latina y el Caribe (orden de 0,68\%), y siendo el umbral sugerido de la UNESCO y países de la OCDE del 1\%.

Tabla 1

Gasto total en $I+D$ en relación al PIB de Paraguay (2008)

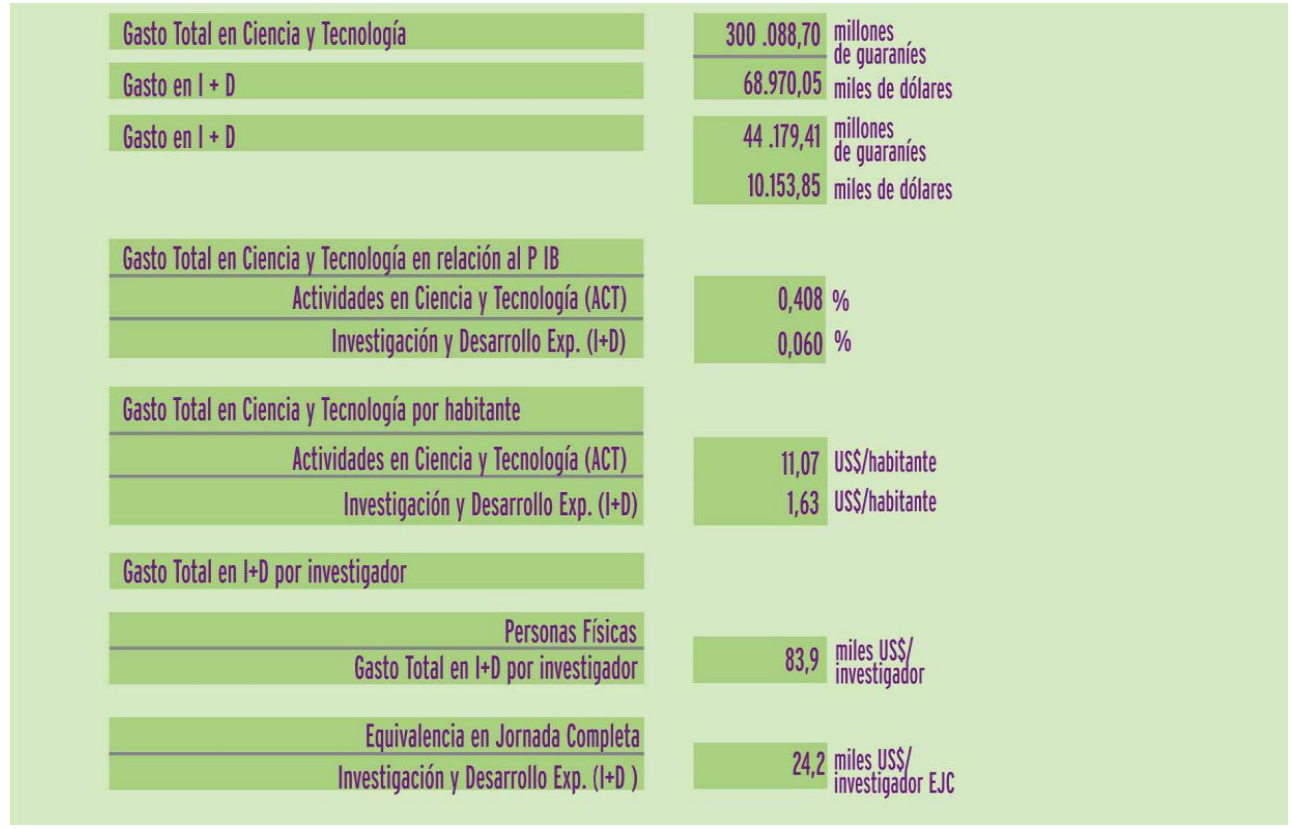

La mayor parte de los esfuerzos en $\mathrm{I}+\mathrm{D}$ se enfoca a la investigación aplicada, al sector publico y agropecuario, relacionado este ultimo con la actividad preponderante de Paraguay. En el sector de las disciplinas científicas, Psicología apenas llega al $0,26 \%$ en su inversión en ciencia y tecnología (Tabla 2 y 3 ).

Los recursos humanos (investigadores), si bien en su mayoría pertenecen al sector de ciencias humanas y sociales, la mayor parte de los recursos financieros se destina al sector agropecuario (46,11\% contra un $11,38 \%)$, como también el objetivo socioeconómico al cual apuntan los trabajos de investigación realizados en Paraguay, tanto a nivel universitario, publico, privado o no gubernamental (Figuras 1 y 2).

Los indicadores de la Educación Superior $(2005,2008)$ se orientan de otra manera: alto predominio de egresados en pregrado versus postgrados, y en las carreras sociales (4337 personas $-62,0 \%$ ), por el contrario, el bajo predominio en las agrarias (300 personas $-4,2 \%$ ), que justamente son a las que destinan el mayor esfuerzo económico (Figuras 3 y 4).

Y se observa un escaso esfuerzo de las universidades en la
I+D, lo que la sitúa en un modelo transmisor de conocimientos y no generador de los mismos, los índices de investigación básica comparados con los propios de investigación aplicada, lo expresan claramente (Figura 5).

Los datos reportados, si bien relevan información general, este contexto macro es extrapolable al estado de desarrollo de la formación y producción científica en Psicología.

\section{Postgrados en psicología o en áreas interdisciplinares}

Los Postgrados en Psicología han sido esporádicos, de cuestionable calidad y de escasa trascendencia en el contexto académico y profesional, no cuentan con líneas de investigación planteadas a nivel institucional, en cuanto a formación científica en: básica, aplicada y tecnológica. Los alumnos tienen libertad de elección de temas para la realización de sus tesis.

A nivel formal son parte de las políticas educativas de Educación Superior, desde inicios de la carrera de pregrado (licenciatura) en Psicología en la década del 1970, en las dos universidades mas importantes que imparten la disciplina psicológica. 
Tabla 2

Gasto en I+D de Paraguay (2008) por Tipo de Actividad, Sector de Financiación, Ejecución y Objetivos Socioeconómicos

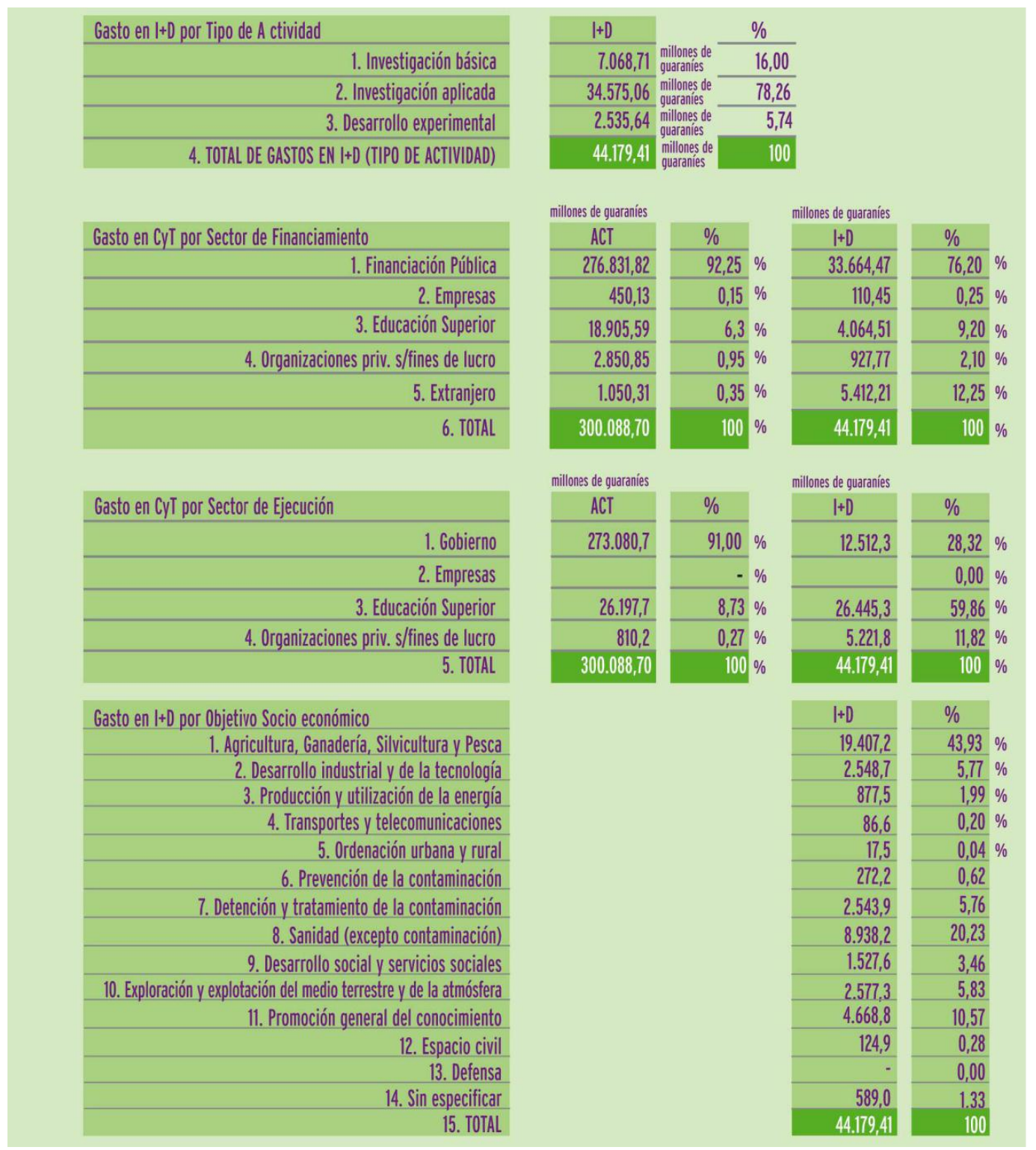

Tanto UCA (1970) como UNA (1976) cuentan estatutariamente con reglamentación para ofrecer y reconocer títulos de post grados a nivel de doctorado. La UNA ha dictado cursos de doctorado en Psicología y actualmente imparte Maestrías. Los titulados en ambos grados son escasos, aproximadamente 20 egresados locales con titulo. Los doctorados no se ofertan en la actualidad.

Recientemente, se "recrea" la Dirección General de Postgrado e Investigación, y los Reglamentos de Doctorado, Maestría y Especialización de la UCA (6/Mayo/10). Los cursos entrarán a regir a partir de 1/Enero/2011

Pero antes de esta fecha, ya existían estatutariamente instituidos, mas no impartidos con regularidad los cursos de Doctorado en la UCA.

Se registran los primeros cursos de Doctorado en Ciencias Sociales en el periodo (1998-2000) a pedido de profesionales de diversas disciplinas de pregrado de la Facultad de Filosofía y Ciencias Humanas-UCA: Derecho, Filosofía, Pedagogía, Psicología y Sociología. Se dictamina la presentación de la tesis de grado y obtención del titulo, como específica, en cada disciplina del egresado. A la fecha de dicho curso, dos doctorandos han obtenido su titulo, uno en Sociología y otra en Psicología.

A inicios del año 2000 se hizo cada vez más fuerte la demanda de apertura de cursos de postgrados en el área de las ciencias sociales, específicamente en el ámbito educativo, tanto de la gestión y de la investigación. Ello estaba estrechamente vinculado con el proceso de reformas educativas de los distintos niveles y del sistema educativo paraguayo.

La Facultad de Filosofía de la UNA y de Filosofía y Ciencias Humanas de la UCA, motivadas por la demanda, ofertan diferentes cursos en formación continua a sus egresados y de 
Tabla 3

Gasto en I+D de Paraguay (2008) por Campo y Disciplina Cientifica

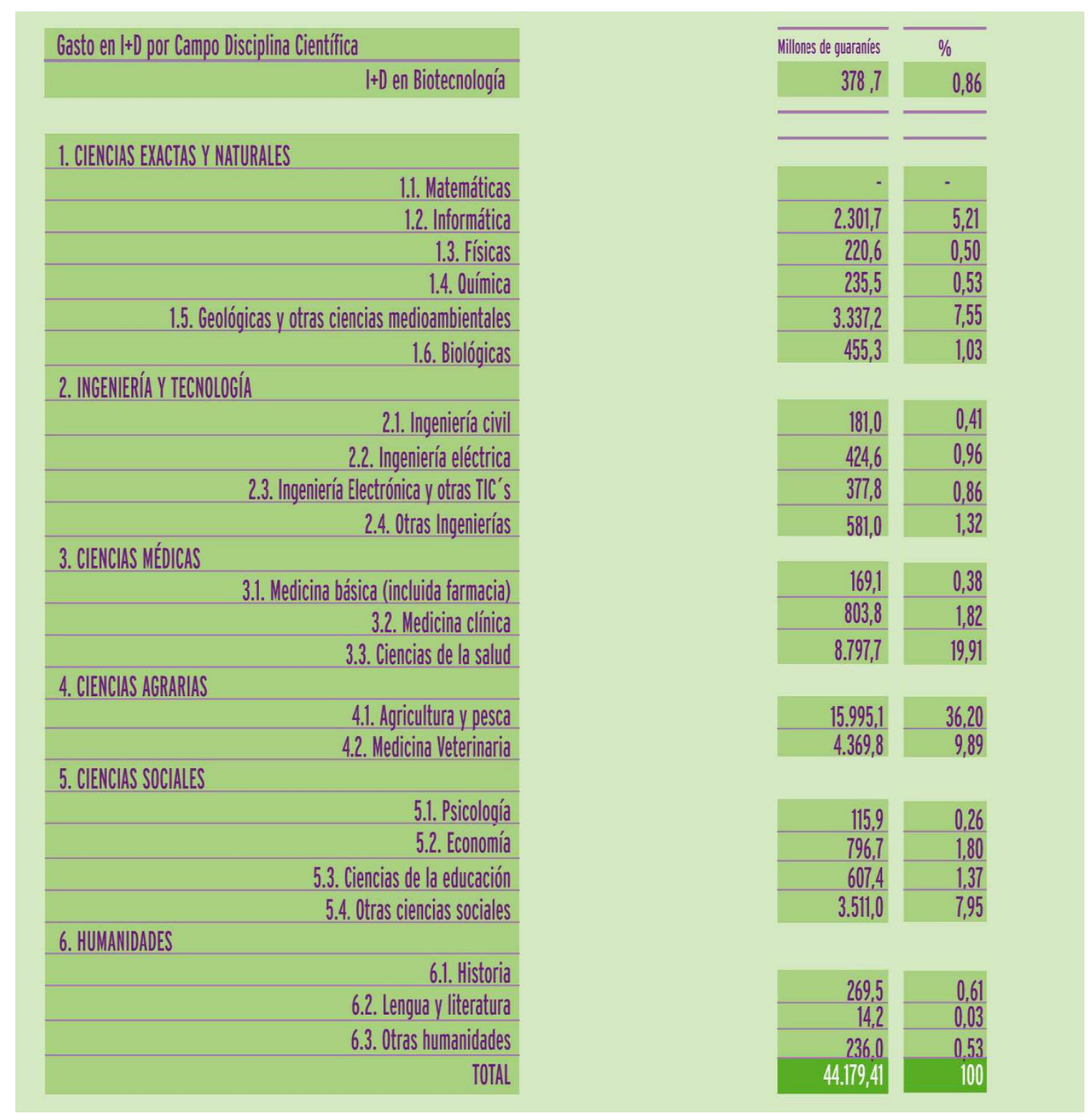

otras instituciones de educación superior.

En el año 2004, se crea el Centro de Postgrado e Investigación, como una unidad académica dependiente de la Facultad de Filosofía y Ciencias Humanas (FFCH-UCA). La FFCH ya contaba con cursos de Postgrados antes de la creación de este centro. En estos seis últimos años, se dictaron maestrías y doctorados en Educación y en Ciencias Sociales.

Los planes de Estudios desarrollados por este centro fueron los siguientes:

Tres áreas disciplinares para Diplomados. Diplomado en Didáctica Universitaria, Gestión de la Comunicación Institucional y Pedagogía Sociohistórica.

Tres áreas disciplinares para Maestrías. a) En Educación: Maestría en Educación con énfasis en Gestión de Entidades Educativas, Maestría en Educación Superior y Maestría en Educación con énfasis en Investigación Educativa; b) En
Antropología Social: Maestría en Antropología Social; c) En Psicología: Maestría en Neuropsicología.

Un Doctorado en Psicología. Con énfasis en intervención social.

Este último curso de postgrado tiene apertura en el año 2006. La creación del Doctorado en Psicología con énfasis en Diagnóstico e Intervención Psicosocial, en la FFCH de la UCA, también respondió a la demanda de profesores, con grado de licenciatura o de maestría, tanto de UNA como de UCA, que solicitaban los cursos como proyecto académico amplio, flexible y de alto rigor científico, enmarcado en la tesitura compleja de la realidad que hoy vive el Paraguay. El programa contó con una duración de 1.200 horas reloj, de las cuales 500 horas son de investigación certificadas por un tutor y 700 dentro del régimen semanal, mensual o intensivo. Esta carga horaria no incluye la 


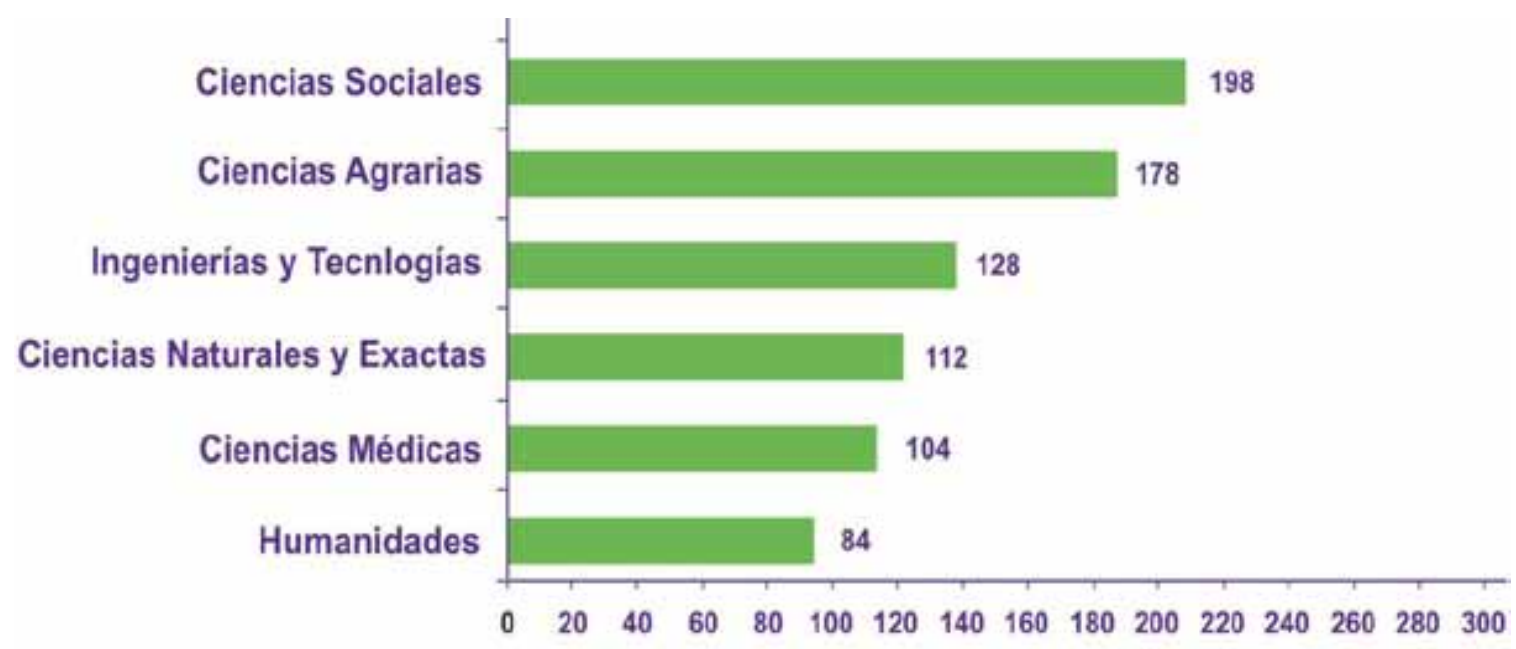

Figural Cantidad de Investigadores distribuidos por área científica.

\section{Cantidad de personas}

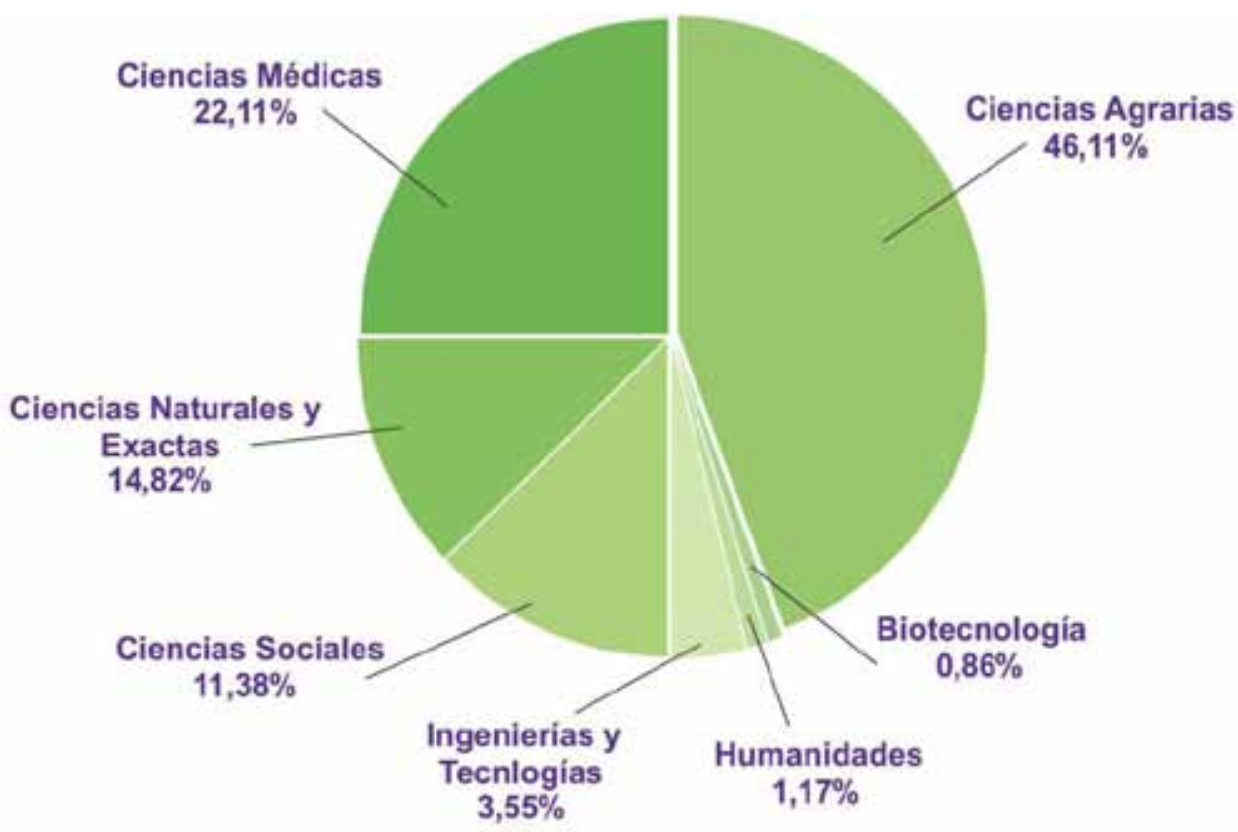

Figura 2. Distribución del gasto en I+D por áreas científicas

Tesis. El titulo que se otorgará será de Doctor (equivalente a PhD) en Psicología, mención en Diagnóstico e Intervención Social, pero a la fecha ningún doctorando lo ha obtenido.

En relación a la UNA se espera que se fortalezca la calidad de los cursos de Maestría actualmente ofrecidos y se vuelvan a ofertar los de Doctorado.

\section{Prospectiva sugerida para la investigación y formación} de postgrado en psicología paraguaya

Las propuestas se plantean, y pueden ser validas, tanto a nivel de contexto general de formación superior e investigación científica, como especifico, para la disciplina psicológica de pre y postgrado, que es nuestro foco de interés en este análisis.

A nivel Macro. Paraguay, a través de sus principales instituciones públicas y privadas, de Educación Superior, debe asumir la Internacionalización del conocimiento, el concepto de cooperación horizontal como estrategia de desarrollo académico.

Se deben desarrollar y aplicar efectivamente las políticas aprobadas a nivel universitario para satisfacer la demanda de calidad y cantidad en la formación de recursos humanos en los grados de maestría y doctorado. (Becas, Sabáticos, Convenios 


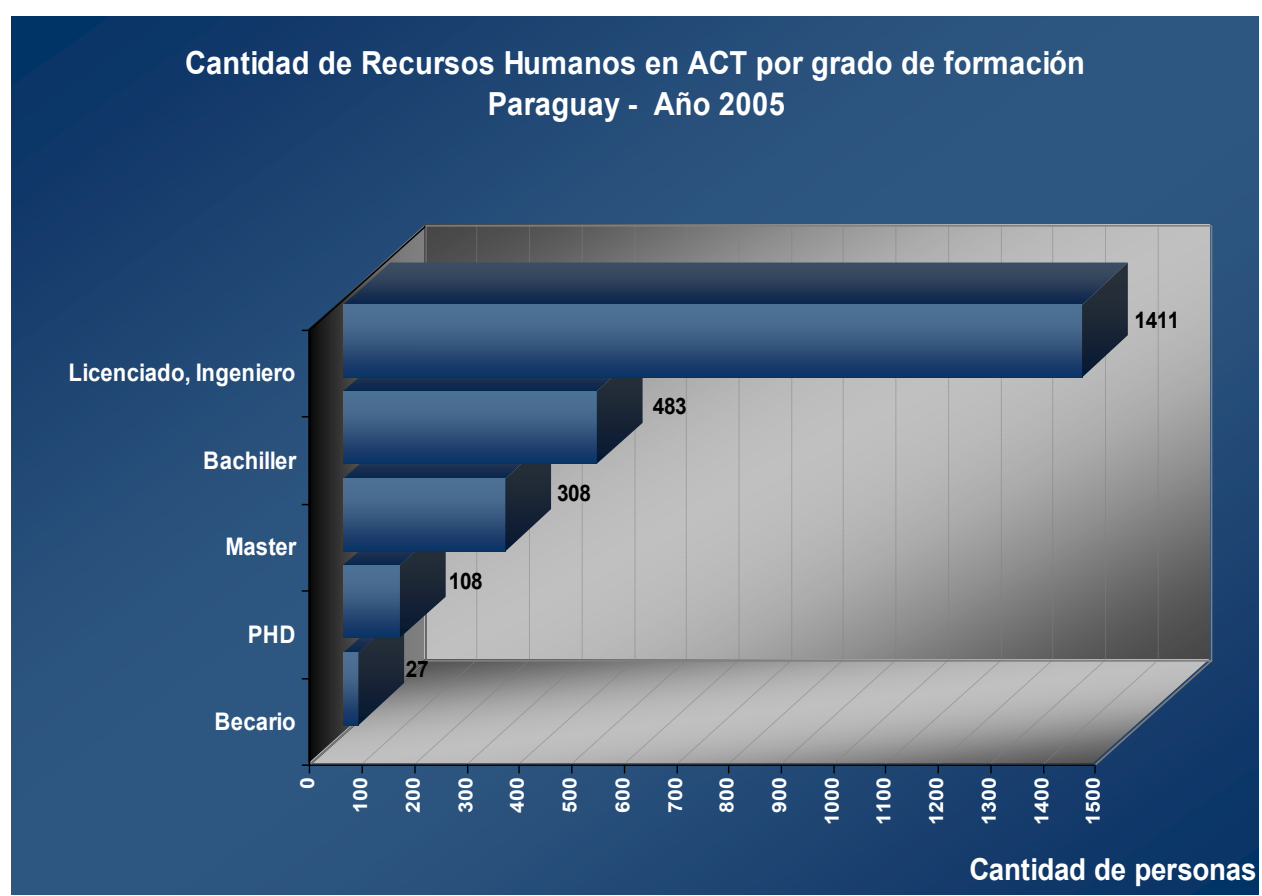

Figura 3. Cantidad de Recursos Humanos en ACT por grado de formación (2005).
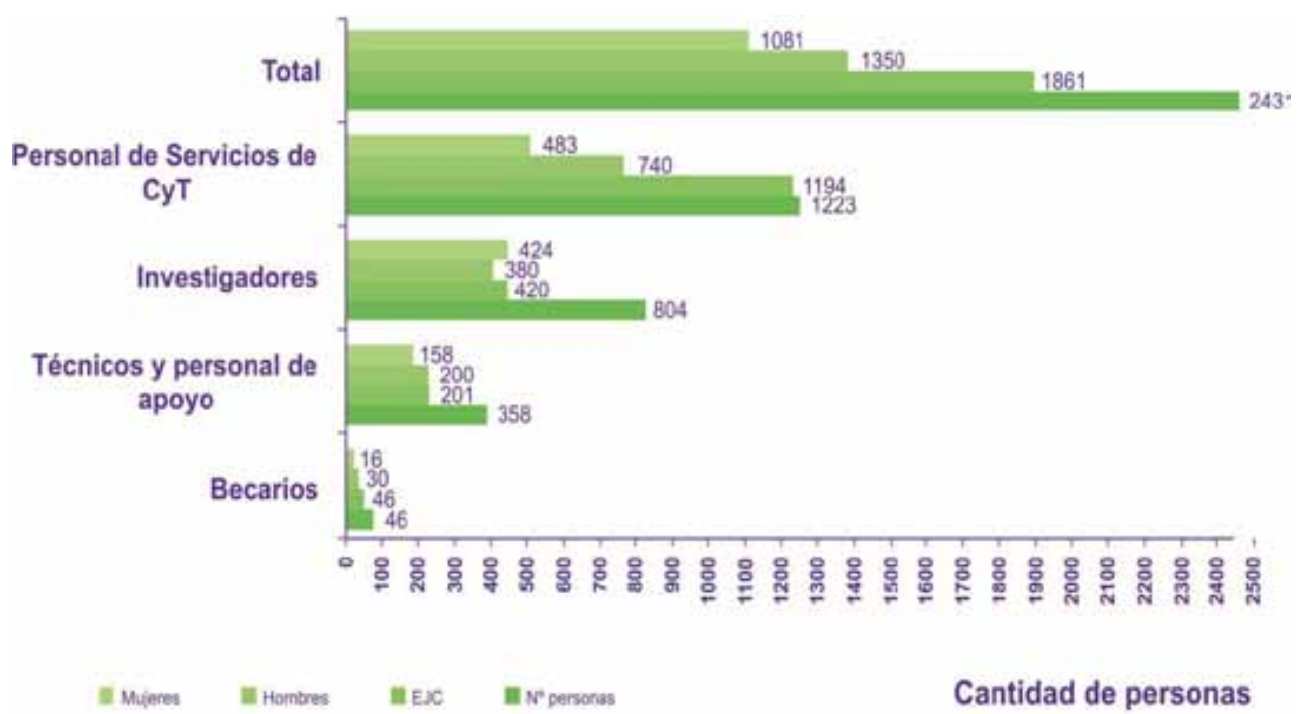

Figura 4. Cantidad de Recursos Humanos en ACT por tipo de actividad y género (2008).

de intercambio entre universidades locales y extranjeras para construir un semillero de recursos calificados).

Asumir el compromiso ante el establecimiento de sistemas internacionales de acreditación de la calidad de la formación superior, y su cumplimiento expedito y significativo, para su concreción en un mayor número de universidades y carreras acreditadas en su calidad, en el breve tiempo posible. La carrera de Psicología a nivel de pregrado, tanto en UNA como en UCA, aun no cuentan con acreditación.

Coexistencia y aplicación de Distintas Metodologías Formativas en un balance necesario ante la mayor oferta de la Enseñanza Virtual (E Learning). Subrayar el compromiso y responsabilidad social de la disciplina, ciencia y profesión psicológica que no puede reducir su formación a los cursos de virtuales.

La Educación Superior del país en el contexto macro de las Universidades Latinoamericanas debe asumir los retos en la formación del postgrado y la producción científica básica, aplicada y tecnológica, del futuro.

Los cambios más previsibles en la modificación de las características de la demanda formativa del postgrado local podrían ser: a) incremento de la demanda de formación continua, en todos sus grados y especializaciones; b) balance entre el modelo tradicional presencial, de densos tiempos y la 


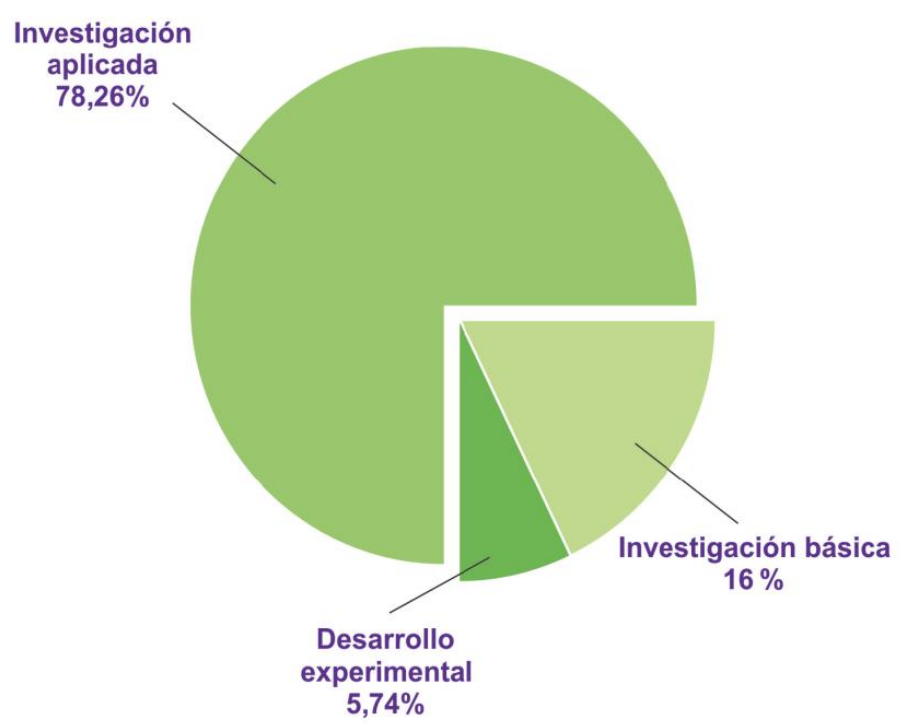

Figura 5. Distribución del gasto (2008), en I+D por tipo de investigación.

actual tendencia a la reducción de los periodos formativos y su modo virtual predominante; c) balance entre la tendencia profesionalizante por competencias técnicas, en el postgrado y la formación científica en sus niveles de básica, aplicada y tecnológica; d) promoción y desarrollo del concepto de Universidad globalizada, (internacionalización de la oferta y la demanda), sin descuidar el desarrollo de enseñanzas vinculadas al contexto socio-geográfico y cultural local, como forma de afrontar una mayor competencia intercultural y preservar la identidad y diversidad propia; e) una estrategia adecuada para superar estos desafíos podría ser el establecimiento de alianzas internacionales entre universidades, la doble titulación, la especialización de los centros formativos, la selección rigurosa de los candidatos evitando la masificación.

A nivel Micro. Crear Redes para formación e investigación en postgrados a nivel local entre las principales universidades que imparten la Carrera de Psicología. A nivel nacional y regional de Pregrado en Psicología, y partir del Tratado de Formación del MERCOSUR, las mallas curriculares mejoraron y actualizaron contenidos, metodologías y sistemas de evaluación.

Promover convenios de formación e intercambios de docentes y estudiantes, fomentando la itinerancia regional y global., en el contexto de las propuestas internacionales del Titulo Europeo (Buela-Casal \& Gutiérrez-Martínez, 2005; Peiro, 2003), y Titulo Latinoamericano de Psicología (Carpintero, 2004; Crespo, 2005, Pelechano, 2005; Sierra \& Bermúdez, 2005).

Urge contar con la aprobación de la Ley de Educación Superior y Colegiado en Psicología, que vele por la formación e investigación de excelencia académica, y el ejercicio profesional competente y ético de la Psicología en el país.

Crear la cultura de investigación y formación en postgrados, e incentivar para ello, la conformación de grupos de investigación intra e inter universitarias para investigación básica, aplicada y tecnológica, la integración de un órgano gubernamental que fomente, defina el perfil de formación de pós-graduados, evalúe y precautele la calidad de los cursos de postgrado en Paraguay.

Fomentar la cultura de la publicación y difusión de los productos de investigación científica generados en pre y postgrado en Psicología, creando revistas científicas de edición periódica en la universidades y asociaciones profesionales, como también una base de datos de las producciones locales, tesis, tesinas, artículos, etc. (como el Centro de Documentación, Investigación y Difusión de la Cátedra de Psicología Experimental de la Carrera de Psicología-UCA - CDID-Eureka).

Llevar en cuenta los saberes populares, los usos y costumbres que hacen a la idiosincrasia paraguaya a la hora de desarrollar contenidos de las mallas curriculares, áreas temáticas de investigación para los PRE y postgrados, buscando conocer, definir y servir a una psicología paraguaya.

Fomentar la investigación aplicada dirigida a las áreas de mejoramiento de la calidad de vida de la población (estilos de vida saludables; entrenamiento en habilidades de prevención del stress; solución y mediación de conflictos, violencia y formas de maltrato diversas; psicología educativa para erradicar todo tipo de discriminación por raza, clase social, género, ideas, etc.; refuerzo de patrones de convivencia y urbanidad ciudadanas, como cuidado del medio ambiente, reciclado, uso racional de las energías, redes de cooperación vecinal para la seguridad, etc.).

Incentivar proyectos específicos de investigación que puedan ser estratégicos para el desarrollo social, económico o cultural del país.

Trabajar sobre Proyectos enfocados al área rural, desprotegida en materia de servicios psicológicos.

Finalmente, estas sugerencias no pretenden ser exhaustivas y exclusivas, se necesita que se fortalezcan los procesos de construcción colectiva entre estudiantes, educadores, investigadores y profesionales de la Psicología paraguaya, para fortalecerla y mejorar la calidad de su formación científicaprofesional. 


\section{Referencias}

Buela-Casal, G., \& Gutiérrez-Martínez, O. (2005). Hacia el título europeo de psicología. Revista Mexicana de Psicología, 22, 243-252.

Carpintero, H. (2004, julio). La Psicología en Iberoamérica. Del pasado al futuro. Conferencia presentada en el IV Congreso Iberoamericano de Psicología, Santiago de Chile. Resumen recuperado de http://www.cop.es/infocop/ vernumero.asp?id $=1145$

Consejo Nacional de Ciencia y Tecnología. (2002). Informe del Consejo Nacional de Ciencias y Tecnologías: Politica Nacional de Ciencia y Tecnología. Recuperado de www.conacyt.org.py

Consejo Nacional de Ciencia y Tecnología. (2010). Estadísticas e Indicadores de Ciencia y Tecnología de Paraguay 2008. Recuperado de www.conacyt.org.py

Coppari, N. B. (1997a) La Investigación en el Ámbito Universitario: Desarrollo Versus Improductividad. Asunción: EDUNA.

Coppari, N. B. (1997b). La Investigación en el Ámbito Universitario: Desarrollo Propuestas de Solución. Asunción: EDUNA.

Coppari, N. B. (2002). Formación científica actual del psicólogo paraguayo en el ámbito universitario. Asunción: FFCH-UCA.
Crespo, I. (2005). La cooperación en Educación Superior: la Red Iberoamericana para la Acreditación de la Calidad (RIACES). Revista Mexicana de Psicología, 22, 345-353.

Duarte Masi, S. (2005). Ciencia, Tecnología y Sociedad: I+D en Paraguay [Presentación Electrónica]. Asunción: CONACYT.

Leyn. 1.028. (1997, 31 de enero). General de Ciencia y Tecnología. Recuperado de http://www.pj.gov.py/ebook/sitios/Libros/Marco_Normativo_Regulatorio_ de_la_Sociedad_de_la_Informacion_en_el_Paraguay.pdf

Peiró, J. M. (2003). La enseñanza de la psicología en Europa: un proyecto de titulación europea. Papeles del Psicólogo, 86, 25-33.

Pelechano, V. (2005). Marco general con una propuesta de contenidos mínimos en psicología. Revista Mexicana de Psicología, 22, 217-223.

Sierra, J. C., \& Bermudez, M. P. (2005). Hacia el título iberoamericano de psicología: análisis de los programas docentes de las carreras de psicología. Revista Mexicana de Psicología, 22, 224-242.

Stufflebeam, D. L., Foley, W. J., Gephart, W. J., Guba, E., Hammond, R. L., Merriman, H. O., \& Provus, M. M. (1981). Educatio-evaluation and decisionmaking in education. Itasca: Peacock.

Norma B. Coppari, doctora en Psicología por Universidad Católica de Asunción (UCA), es docenteinvestigadora en la Universidad Nacional de Asunción. Correspondencia: Francis Morices nํㅗㄴ 590, Esq/César Medina, Villa Aurelia, Asunción, Paraguay. Correo electrónico: norma@tigo.com.py 